\title{
Brown and beige fat in humans: thermogenic adipocytes that control energy and glucose homeostasis
}

\author{
Labros Sidossis ${ }^{1}$ and Shingo Kajimura² \\ 'Metabolism Unit, Shriners Hospitals for Children, Departments of Internal Medicine and Surgery, University of Texas, Medical Branch at Galveston, Texas, USA. ${ }^{2}$ UCSF Diabetes Center \\ Department of Cell and Tissue Biology, UCSF, San Francisco, California, USA.
}

\begin{abstract}
Brown adipose tissue (BAT), a specialized fat that dissipates energy to produce heat, plays an important role in the regulation of energy balance. Two types of thermogenic adipocytes with distinct developmental and anatomical features exist in rodents and humans: classical brown adipocytes and beige (also referred to as brite) adipocytes. While classical brown adipocytes are located mainly in dedicated BAT depots of rodents and infants, beige adipocytes sporadically reside with white adipocytes and emerge in response to certain environmental cues, such as chronic cold exposure, a process often referred to as "browning" of white adipose tissue. Recent studies indicate the existence of beige adipocytes in adult humans, making this cell type an attractive therapeutic target for obesity and obesity-related diseases, including type 2 diabetes. This Review aims to cover recent progress in our understanding of the anatomical, developmental, and functional characteristics of brown and beige adipocytes and discuss emerging questions, with a special emphasis on adult human BAT.
\end{abstract}

\section{Historical perspective on human BAT}

Brown adipose tissue (BAT) evolved as a natural defense system against hypothermia in mammals. BAT is composed of a specialized form of adipocytes that contain numerous lipid droplets (multilocular lipids) and large mitochondria with the BAT-specific protein, uncoupling protein 1 (UCP1). UCP1 is localized in the inner membrane of mitochondria and diminishes the proton gradient by uncoupling cellular respiration and mitochondrial ATP synthesis. Activation of UCP1 in response to cold exposure or food intake results in increased glucose and free fatty acid (FFA) oxidation in order to sustain high levels of uncoupled respiration within BAT, that is, mitochondrial respiration that leads to heat generation.

While the long-standing, prevailing view was that BAT exists only in small mammals and newborns $(1,2)$, evidence for the existence of BAT depots in adult humans dates back to the early 1900s. Anatomists suggested the presence of a paired "gland" on the shoulder and the side of the neck of adult humans in 1908 (3) and reported on its morphological similarities with the interscapular BAT depot in animals (4). Although some reports from outdoor workers and postmortem studies indicated that BAT may still be present in human adults even later in life (5-7), the scientific momentum in BAT research dwindled due to the lack of methodological approaches to quantify BAT and the scarcity of evidence that human adults possess significant amounts of functional BAT (8). The first evidence supporting the notion that active BAT is present in adult humans began to emerge in 2002, using ${ }^{18} \mathrm{~F}$-fluoro2-deoxy-D-glucose $\left({ }^{18} \mathrm{~F}-\mathrm{FDG}\right)$ positron emission tomography computed tomography scans (referred to herein as ${ }^{18} \mathrm{~F}-\mathrm{FDG}-\mathrm{PET} / \mathrm{CT}$

Conflict of interest: The authors have declared that no conflict of interest exists. Reference information: / Clin Invest. 2015;125(2):478-486. doi:10.1172/JCI78362. scans). Radiologists observed an increased symmetrical "tumorlike" glucose uptake in supraclavicular adipose tissue depots, which was suggested to represent BAT (9). Soon after, numerous retrospective studies described the prevalence and localization of the increased adipose tissue ${ }^{18} \mathrm{~F}-\mathrm{FDG}$ uptake, along with possible predictors (age, gender, outdoor temperature; refs. 10-12) and possible interventions (warming patients, propranolol administration) to minimize the prevalence of those false-positive images intended for diagnostic purposes $(12,13)$. Furthermore, the association between the ${ }^{18} \mathrm{~F}-\mathrm{FDG}$ uptake in the supraclavicular adipose tissue and ambient temperature suggested a potential functional role for adult human BAT (14). Landmark papers by several independent groups clearly demonstrated the functional and molecular evidence that active BAT depots are present in adult humans and can be readily activated when exposed to mild cold (15-19). Now a number of prospective studies have reported that many, if not all, adult humans possess metabolically active BAT, and its mass shows an inverse correlation with BMI and adiposity $(15,16$, 19-21). While the presence of BAT in humans is now undisputed, many outstanding questions need further investigation. What are the molecular and functional characteristics of human BAT? How is BAT mass and function regulated? What is the physiological and clinical impact of BAT? We aim to review recent progress in these topics and discuss emerging questions in the field of BAT biology, with a special emphasis on human BAT.

\section{Anatomical features of brown and beige adipocytes}

Based on the developmental and anatomical features, at least two types of thermogenic adipocytes exist in mammals: classical brown adipocytes and beige adipocytes (also known as brite adipocytes). As summarized in Figure 1, classical brown adipocytes 
A

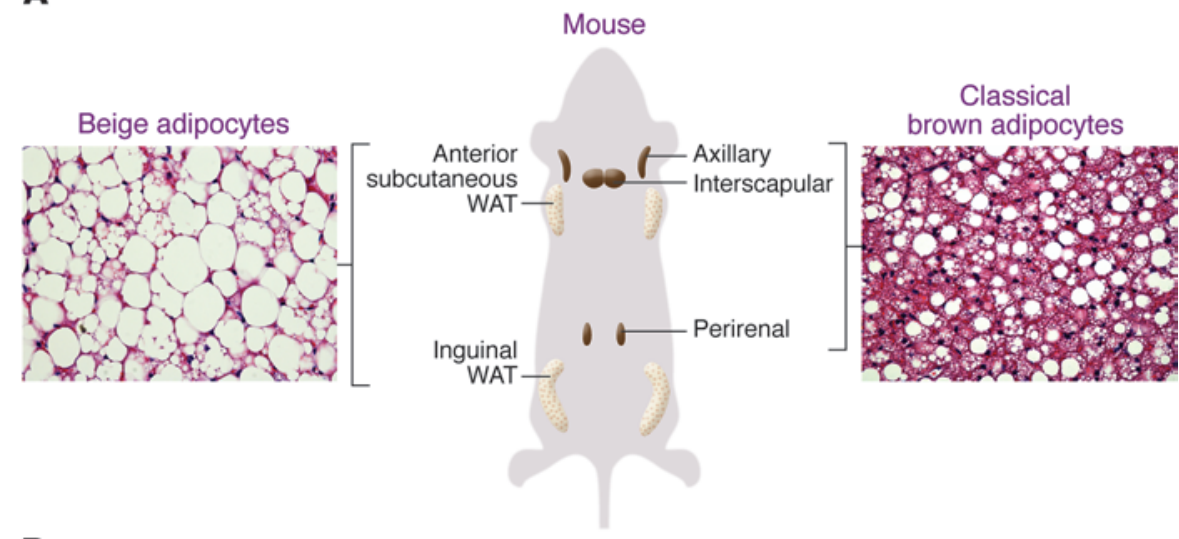

Figure 1. Anatomical locations of thermogenic adipocytes in mice and humans. (A) In rodents, interscapular BAT and perirenal BAT are composed of classical brown adipocytes. Beige adipocytes are largely found in subcutaneous WAT (anterior and inguinal WAT) and are highly recruitable (a process referred to as "browning" of WAT) by certain external cues. (B) Adult human BAT is located mainly in the cervical, supraclavicular, and paravertebral regions. In infants, BAT is found in the interscapular and perirenal regions.

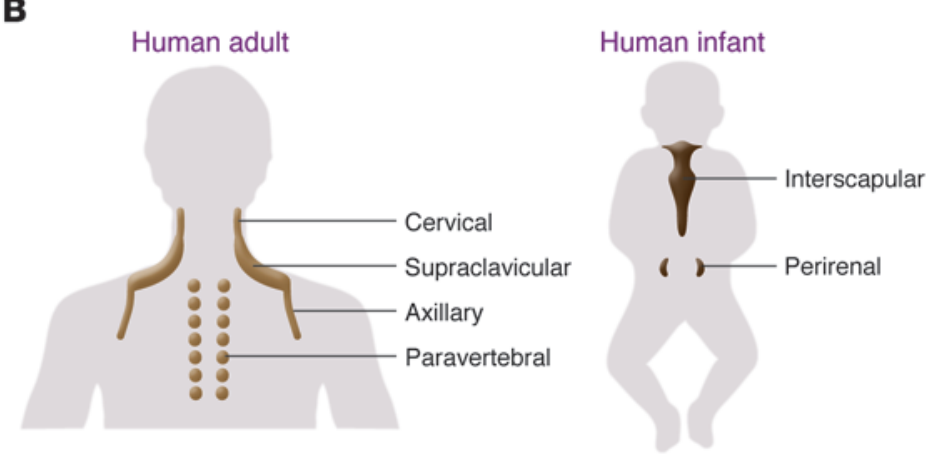

are found in the major dedicated BAT depots of rodents, such as in the interscapular, perirenal, and periaortic regions. Since BATmediated thermogenesis is crucial for maintaining body temperature in infants, formation of the BAT depots (i.e., classical brown adipocyte development) is largely completed during the prenatal stage in order to produce heat right after birth through nonshivering thermogenesis. On the other hand, beige adipocytes exist mainly in subcutaneous white adipose tissue (WAT) of rodents. Beige adipocytes sporadically reside within WAT, and their development is dramatically induced in response to certain external cues, such as chronic cold exposure, exercise, and long-term treatment with PPAR $\gamma$ agonists (e.g., rosiglitazone). This phenomenon, which occurs in the postnatal stages, is often called "browning" of WAT (22-24). When stimulated by such external cues, beige adipocytes express UCP1 protein at a similar level to classical brown adipocytes and exhibit UCP1-dependent thermogenic capacity $(25,26)$.

In humans, classical brown adipocytes exist in the interscapular BAT of infants (27). While the BAT depots of rodents remain throughout life, human brown adipocytes in the interscapular region gradually disappear with age (7). In adults, ${ }^{18} \mathrm{~F}-\mathrm{FDG}-\mathrm{PET} /$ CT scans have identified active BAT depots in the cervical, supraclavicular, axillary, and paravertebral regions. From an evolutional point of view, BAT around the neck of adult humans and nonhuman primates may evolve to protect the brain by warming up the blood supplied to the brain.

The two types of thermogenic adipocytes are also distinct in their cellular heterogeneity within adipose tissues. The interscapular and perirenal BAT depots are largely composed of classical brown adipocytes with multilocular lipid droplets and separated from WAT by a layer of connective tissue $(27,28)$. In contrast, beige adipocytes in WAT exist together with unilocular white adipocytes, without any separation by a distinct later of connective tissue.

Under cold stimulation, clusters of beige adipocytes emerge in the area in which tyrosine hydroxylase-expressing (noradrenergic) nerve fibers are densely innervated $(28,29)$, as norepinephrine released from the sympathetic nerves is a powerful stimulator of WAT browning. Intriguingly, adult human brown adipocytes are also sporadically found together with white adipocytes, and the density is well correlated with the number of tyrosine hydroxylase-positive noradrenergic nerves $(27,30)$.

\section{Developmental origins and plasticity of brown and beige adipocytes}

Brown and beige adipocytes share many biochemical characteristics, including enriched mitochondria, multilocular lipid droplets, and expression of UCP1. However, they originate from distinct cellular lineages. As shown in Figure 2A, classical brown adipocytes originate from a subpopulation of dermomyotomes that can also give rise to skeletal muscle. A lineage-tracing study found that engrailed-1-expressing (En1-expressing) cells in the central dermomyotome differentiate to BAT, skeletal muscle, and dermis (31). Nearly the entire population of classical brown adipocytes in the interscapular BAT arises from precursors that express $M y f 5$, a gene known to be expressed in committed skeletal muscle precursors (32). A pulse-chase tracing study used another myogenic marker, Pax7, and showed that the divergence of myoblasts and brown adipocyte progenitors occurred between embryonic days 9.5 and 11.5 in mice (33). As an independent line of evidence, the transcriptional profile of classical brown adipocytes resembles that of skeletal muscle cells $(34,35)$. Similarly, the mitochondrial proteomic profile of BAT is more related to that of skeletal muscle than that of WAT (36). 
A

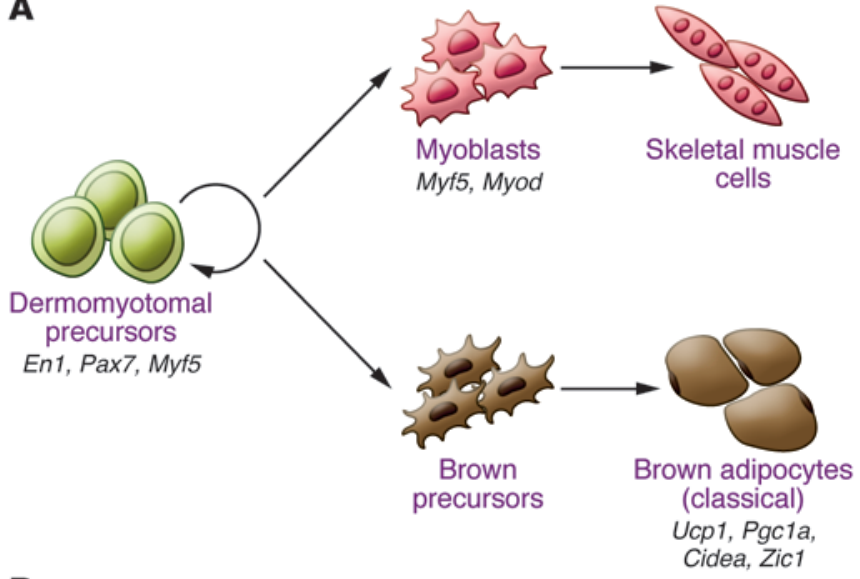

B

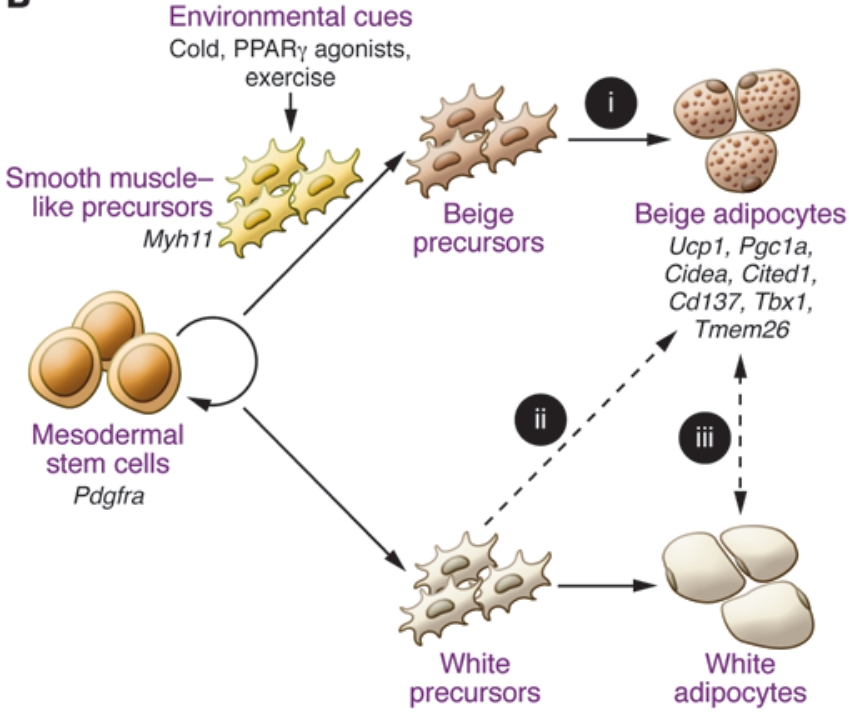

The cellular origin of beige adipocytes has not been completely elucidated. UCP1-expressing beige adipocytes in the epididymal WAT arise through the proliferation and differentiation of precursors that express platelet-derived growth factor receptor $\alpha$ (PDGFR $\alpha$ ), CD34, and spinocerebellar ataxia type 1 (SCA1) (37). Beige adipocytes in the inguinal WAT were reported to derive from Myf5-negative precursors (32), whereas Sanchez-Gurmaches et al. showed that a subset of beige adipocytes in multiple WAT depots, such as anterior subcutaneous and perigonadal depots, can originate from Myf5-positive cells (38). Furthermore, a recent paper by Spiegelman's group showed that a subset $(10 \%-15 \%)$ of UCP1-positive beige adipocytes, but not classical brown adipocytes, arises from precursors that express $M y h 11$, one of the most selective markers for smooth muscle cells (39). Collectively, these results indicate that beige adipocytes have distinct cellular origins from classical brown adipocytes and are composed of heterogeneous cell populations.

Another intriguing yet enigmatic issue is the cellular plasticity of beige adipocytes. Several studies indicate that mature white adipocytes may directly convert into beige adipocytes (i.e., transdifferentiation) in vivo. For example, adipocytes at an intermediate state between white and beige that contain "paucilocular" lipid droplets have been observed during the process of cold adaptation
Figure 2. Developmental origins of brown and beige adipocytes. (A) Classical brown adipocytes originate from a subset of dermomyotomes that express En1, Pax7, and Myf5. Classical brown adipocytes express several markers, including Ucp1, Pgc1a, and Cidea, and classical brownselective markers, such as Zic1. (B) Beige adipocytes in WAT originate from MYF5-negative PDCFR $\alpha$-positive precursors of mesodermes. A subset of beige adipocytes arises from MYH11-positive smooth muscle-like precursors. Beige adipocyte differentiation is induced by environmental cues, such as chronic cold exposure, exercise, and PPAR $\gamma$ agonists. Beige adipocytes express several markers, including Ucp1, Pgc1a, and Cidea, and beige-selective markers, such as $[d 137, T b \times 1$, Tmed26, and Cited1. These cells may be derived from (i) defined beige precursors, (ii) directed differentiation from white precursors, or (iii) transdifferentiation from white adipocytes in a differentiated state. The dashed lines represent hypothetical models that require further investigation.

(40). A large part of the newly formed beige adipocytes in subcutaneous WAT is negative for BrdU or Ki67, such that mitotic proliferation is not required for the cold-induced beige adipocyte differentiation $(37,41,42)$. Furthermore, a recent genetic lineage-tracing study showed that beige adipocytes converted into mature white adipocytes after 5 weeks of warm adaptation. These white adipocytes can reconvert into beige adipocytes after additional cold stimulation (43). On the other hand, Scherer's group performed pulse-chase fate-mapping experiments of mature adipocytes and found that the majority of newly developed beige adipocytes stem from de novo differentiation of precursors in subcutaneous WAT (44). Hence, the transdifferentiation hypothesis still needs to be critically tested by monitoring the life cycle of beige adipocytes at a single-cell resolution.

\section{Cellular identity of adult human BAT - is human BAT recruitable?}

Recent studies have identified several genes that are preferentially expressed either in classical brown adipocytes or in beige adipocytes in rodents $(26,39,45,46)$. Based on the expression profiles of these genes, the interscapular BAT of human infants is composed of adipocytes expressing a gene signature of classical brown adipocytes (27). On the other hand, BAT in the supraclavicular regions of infants and in adult humans expresses high levels of beige adipocyte-enriched genes, including Tbx1, Cd137, Tmem 26, and Cited $1(26,27,45)$. These findings imply that adult human BAT depots may possess beige-like adipocytes; however, more recent studies report that adult human BAT expresses classical brown adipocyte-selective markers $(47,48)$. This discrepancy appears to be primarily due to the high heterogeneity within human BAT. Indeed, Cypess et al. reported that gene signatures of human BAT vary depending on the depth of BAT in the neck (48). Importantly, UCP1-positive adipocytes sporadically colocalize with white adipocytes and other cell types within the BAT depots, and molecular analyses of biopsied adipose tissue samples could be highly confounded by potential contamination of UCP1-negative cells. Hence, it is important to understand the nature of adult human brown adipocytes in homogeneous cell populations.

To address this, we have recently isolated clonal UCP1-positive adipocytes from adult human BAT. The isolated adipocytes exhibit molecular and functional characteristics of bona fide brown adipocytes based on their expression of UCP1 and thermo- 


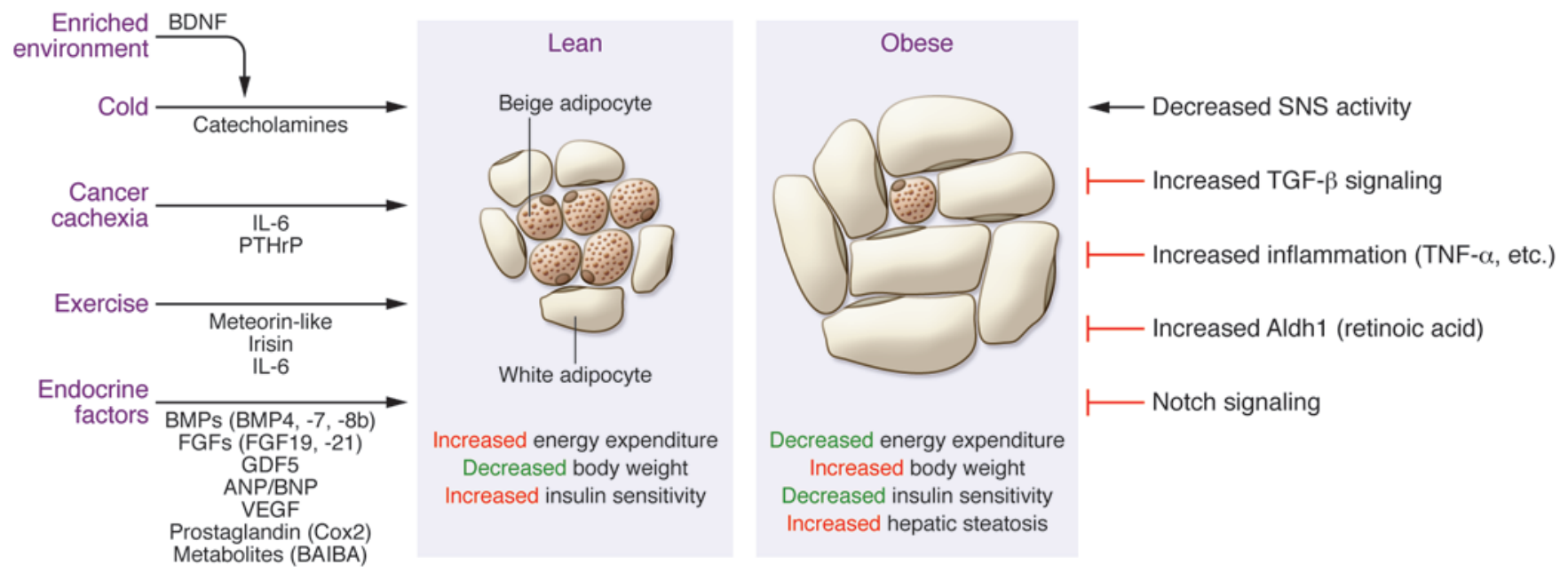

Figure 3. External and internal factors that regulate beige adipocyte development under lean and obese conditions. Beige adipocyte development is induced by the indicated stimuli and mediated by the listed endocrine factors. This process is associated with increased energy expenditure, reduced body weight, and improved insulin sensitivity. Under obese conditions, beige adipocyte development is impaired through multiple factors, including but not limited to reduced sympathetic nervous system (SNS) activity and increased activation of signaling pathways that inhibit beige adipocyte development. Impaired beige adipocyte development is associated with reduced energy expenditure, increased body weight, reduced insulin sensitivity, and development of hepatic steatosis.

genic capacity. Genome-wide analyses based on RNA sequencing indicate that the cloned human brown adipocytes possess molecular signatures that resemble murine beige adipocytes, rather than classical brown adipocytes. These data suggest that adult human BAT is mainly, if not all, composed of beige-like "recruitable" thermogenic adipocytes. This view is supported by recent works on chronic cold acclimation-recruited new BAT in the supraclavicular region of adult humans who did not possess detectable BAT before treatment (49-51). Importantly, the emergence of newly recruited BAT was in parallel with an increase in cold-induced energy expenditure (49-50) or an improvement in postprandial insulin sensitivity (51). In addition, the BAT mass was significantly higher in winter than in summer (19). These results indicate that adult human BAT possesses a high plasticity and can be recruited by external cues, even in populations that lose active BAT depots. Cellular identities of UCP1-expressing adipocytes in other adipose depots, such as in the perirenal and paravertebral regions and in the subcutaneous WAT depots, await further investigation.

\section{How are beige adipocytes recruited by external cues?}

Because of its inducible nature and the relevance to adult humans, beige adipocytes have recently attracted much attention as a new therapeutic target for obesity. From a basic scientific view, beige adipocytes also provide a fascinating experimental system through which our understanding of how environmental changes affect cell fate specification and maintenance can be increased. While many cell fate determination processes are precisely regulated by genetic and internal cues, the fate of beige versus white adipocytes is a unique example in which external cues, such as temperature and nutrition, are dominant determinants. A number of positive and negative regulators of brown/beige adipocyte development have been identified, as summarized by recent reviews (22-24, 52). Here, we focus on new players, with a special emphasis on endocrine factors that mediate the external cue-induced beige adipocyte development and function (Figure 3).

Chronic cold exposure. Classically, norepinephrine released from the sympathetic nerve terminals activates the BAT thermogenic program via PKA and p38-MAPK signaling, followed by the production of FFA by lipolysis for UCP1-mediated proton uncoupling. These pathways also powerfully stimulate the expression of $U c p 1$ through the phosphorylation of key transcriptional activators, including PPAR $\gamma$ coactivator $1 \alpha$ (PGC1 $\alpha$ ), cAMP response element-binding protein (CREB), and activating transcription factor 2 (ATF2) (53). Chawla's group identified a new population of regulatory cells that mediate cold response: alternatively activated (type 2/M2) macrophages. When activated by eosinophils via IL-4 and IL-13 signaling, M2 macrophages are recruited to subcutaneous WAT and secrete catecholamines to activate WAT browning $(54,55)$. Mice that lacked IL-4 signaling or catecholamine production selectively in macrophages exhibited severe impairment in cold-stimulated beige adipocyte development. Conversely, IL-4 administration was sufficient to induce WAT browning and reduce diet-induced body weight gain (55).

Environmental enrichment. An enriched living environment with complex physical and social stimuli increases energy expenditure. Mice in an enriched environment are more resistant to diet-induced obesity, in part, through WAT browning. Hypothalamic brain-derived neurotrophic factor (BDNF) mediates the environment-associated activation of the sympathetic nervous system and induces beige adipocyte differentiation. Hypothalamic overexpression of BDNF sufficiently reproduces the enrichment-induced WAT browning and results in a lean phenotype (56).

Exercise. Certain types of exercise have been shown to induce WAT browning and increase energy expenditure $(57,58)$. PGC1 $\alpha$ is a key transcriptional coregulator that mediates a number of physiological changes in the skeletal muscle during exercise, including mitochondrial biogenesis and an activation of the oxidative 
phosphorylation gene program (59). Two novel myokines, irisin and meteorin-like (METRNL), are upregulated by the exerciseinduced PGC1 $\alpha$ pathway and activate beige adipocyte development $(57,60)$. Upregulation of circulating irisin has also been reported in adult humans during cold exposure (61). Irisin induces the expression of beige adipocyte-selective genes in a cell-autonomous manner, whereas METRNL promotes an eosinophil-dependent activation of M2 macrophages and induces WAT browning. Additionally, a major myokine, IL-6, has been shown to activate beige adipocyte development and also be required for exerciseinduced WAT browning in mice (62). Intriguingly, lactate, a major product of anaerobic glycolysis in skeletal muscle during exercise, has been shown to promote WAT browning (63). The lactateinduced WAT browning is mediated by a change in intracellular redox state $\left(\mathrm{NADH}-\right.$ to- $\mathrm{NAD}^{+}$ratio) via the monocarboxylate transporters that drive lactate into the cells. While the physiological significance of lactate on exercise-induced WAT browning awaits further studies, this report identified a new role of lactate on beige adipocyte development and energy homeostasis.

Cancer cachexia. Cachexia is characterized by severe weight loss, chronic inflammation, and muscle/adipose atrophy. Cancer cachexia is often associated with WAT browning and increased energy expenditure. The WAT browning was significantly impaired when an IL-6-deficient tumor was implanted, indicating that IL- 6 acts as a major mediator of cachexia-induced beige adipocyte development (64). Spiegelman and colleagues reported that tumor-derived parathyroid hormone-related protein (PTHrP) promotes WAT browning and increased energy expenditure in a Lewis lung carcinoma model (65). Neutralization of PTHrP in mice blocks WAT browning and protects mice from energy wasting in WAT and skeletal muscle.

Endocrine hormones and metabolites. A number of endocrine regulators for brown/beige adipocytes have been identified. As listed in Figure 3, such factors include BMP4 $(66)$, BMP7 $(67,68)$, BMP8b (69), FGF19 (70, 71), FGF21 (72, 73), growth differentiation factor-5 (GDF5) (74), natriuretic peptides (75), prostaglandins $(76,77)$, $\operatorname{VEGF}(78,79)$, and $\beta$-aminoisobutyric acid (BAIBA) (80). Indeed, these factors are able to increase whole-body energy expenditure, protect animals from diet-induced body weight gain, and improve systemic glucose homeostasis and/or insulin sensitivity.

Under obesity conditions, several negative regulators are reported to inhibit brown/beige adipocytes. For example, TGF- $\beta$ expression is increased under obesity and blocks beige adipocyte differentiation. Conversely, blockade of TGF- $\beta$ signaling by genetic ablation of Smad3, a major mediator of TGF- $\beta$ signaling, or by administration of a TGF- $\beta$-neutralizing antibody enhances WAT browning and protects animals from diet-induced obesity and insulin resistance (81-83). Similarly, depletion of retinaldehyde dehydrogenase (Aldh1), a rate-limiting enzyme that generates retinoic acid (84), or inhibition of Notch signaling (85) promotes WAT browning and increases energy expenditure in rodents.

Transcriptional mediators. While many of the known transcriptional regulators negatively control brown/beige adipocyte development, PR domain-containing protein 16 (PRDM16) and its binding partners, such as CCAAT/enhancer-binding protein- $\beta$ $(\mathrm{C} / \mathrm{EBP} \beta)$, early B cell factor 2 (EBF2), PGC1 $\alpha$, and euchromatic histone-lysine N-methyltransferase 1 (EHMT1), serve as positive regulators. Depletion of such factors disrupts fate determination and/or maintenance of brown and beige adipocytes (32, 86-90). Importantly, cold- and PPAR $\gamma$ agonist-induced WAT browning is substantially impaired in the absence of PRDM16 or EHMT1, indicating that these regulators mediate environment-induced beige adipocyte development $(88,91,92)$.

\section{Genetic contribution to BAT development and obesity}

A series of studies by Kozak's group previously reported a strong inverse association between browning propensity in WAT and genetic susceptibility to diet-induced obesity in rodents (93). Notably, such genetic susceptibility is linked to Ucp1 expression in WAT (i.e., beige adipocytes) but not with that in interscapular BAT (i.e., classical brown adipocytes), highlighting the biological significance of beige adipocytes in the propensity to obesity $(94,95)$.

In humans, genetic links to BAT mass and energy expenditure remain less understood. Polymorphisms in UCP1 (-3826 $\mathrm{A} / \mathrm{G})$ and $\beta 3$-adrendergic receptor (ADRB3) (64 Trp/Arg) are associated with lower resting energy expenditure and decreased cold-induced thermogenesis $(96,97)$. Furthermore the BAT prevalence was significantly lower in older subjects with polymorphisms in the UCP1 and $\beta 3$-adrendergic receptor genes (98). Two recent papers identified human genetic mutations/ variations that affect BAT development and energy metabolism. EHMT1 is a nuclear-localized methyltransferase that is required for brown and beige adipocyte development. Lack of EHMT1 in adipose tissue lowers whole-body energy expenditure and causes obesity, hepatic steatosis, and insulin resistance in mice (88). Notably, $40 \%$ to $50 \%$ of the human patients with EHMT1 haploinsufficiency develop obesity $(99,100)$, indicating the importance of EHMT1 in energy homeostasis in humans. In contrast, deletion of the gene encoding the GPCR leucine-rich repeat-containing GPCR4 (LGR4), promotes WAT browning, in part, by reducing the expression of the retinoblastoma $1(R b 1)$ gene. Lgr4-null mice were protected from diet-induced obesity and insulin resistance (101). Intriguingly, a gain-of-function variant (A750T) of LGR4 is associated with obesity in a Chinese population (101). Given the prevalence of BAT in adult humans, new genetic links to human BAT development and obesity will be identified in the near future.

\section{Metabolic benefits of BAT - whole-body energy and glucose homeostasis}

Evidence from rodent studies clearly demonstrates that BAT plays a significant role in whole-body energy homeostasis, substrate metabolism, and glucose homeostasis. For example, Ucp1 knockout mice under thermoneutral conditions exhibit impaired diet-induced thermogenesis and develop obesity (102). BAT transplantation increases energy expenditure and improves glucose homeostasis (103). Moreover, BAT clears $75 \%$ and $50 \%$ of the total glucose and triglycerides from the circulation, respectively (104). It is important to note that manipulation of beige adipocytes is sufficient to alter energy expenditure and glucose homeostasis in mouse models $(92,105)$. An emerging idea is that generating a new "metabolic sink" for glucose, triglycerides, and possibly other metabolites through promoting beige adipocyte development can 
lead to an improvement in systemic glucose and lipid homeostasis, in addition to an increase in whole-body energy expenditure.

In adult humans, retrospective studies that used ${ }^{18} \mathrm{~F}-\mathrm{FDG}-\mathrm{PET} /$ $\mathrm{CT}$ associated BAT activity with leanness, euglycemia, decreased probability of development of insulin resistance and diabetes mellitus, and an improved lipid profile (16, 106-109). Although the lack of standardized conditions (e.g., ambient temperature, diet) and the inclusion of patients with unknown cancer status may limit the interpretation of these reports, many well-controlled experimental studies have used nonshivering cold exposure and demonstrated the role of BAT on energy expenditure in adult humans. For example, cold-induced thermogenesis was reported to be proportional to BAT perfusion $(110)$ and activity $(15,49)$, even after adjusting for potential confounding factors (111). Other studies examined the effect of cold exposure on energy expenditure in individuals with or without detectable BAT and found that BAT-mediated activation increases energy expenditure by $10 \%$ to $27 \%(20,49,50,112)$. Using a prolonged (5-hour), personalized cold exposure protocol above the individuals' shivering threshold to maximize BAT thermogenesis, it is estimated that 50 to $100 \mathrm{ml}$ of BAT can increase energy expenditure by 150 to $300 \mathrm{kcal}$ a day in healthy men (20). Furthermore, Lee et al. used a rigorous study design and demonstrated that BAT increased diet-induced thermogenesis by $32 \%$ in young men (51).

In addition to the role of BAT in energy expenditure, recent studies have been designed specifically to investigate the role of BAT in glucose metabolism in adult humans. Cold exposure significantly increases glucose disposal in BAT $(110,113)$ but not in other tissues. Obese individuals seem to exhibit a less pronounced response (21). Furthermore, Saito's group reported recently that blood HbA1c, total cholesterol, and LDL cholesterol levels were significantly lower in the BAT-positive group than those in the BAT-negative groups (114). These findings suggest that activated BAT has the ability to clear glucose from circulation; however, it is still debatable whether this response is clinically significant. For example, no differences in whole-body insulin-stimulated glucose disposal were found between individuals with detectable BAT and those with nondetectable BAT (21). Ouellet et al. showed that BAT primarily oxidizes intracellular fatty acids under acute cold exposure, whereas plasma glucose and FFA oxidation by BAT was marginal (113). On the other hand, using stable isotopic tracers and euglycemic-hyperinsulinemic clamps, we reported recently that prolonged (5-hour) but mild cold exposure increased whole-body glucose disposal, plasma glucose oxidation, and insulin sensitivity in men with significant amounts (i.e., over $50 \mathrm{ml}$ ) of BAT. Notably, cold exposure did not affect whole-body glucose metabolism in men who did not have detectable BAT. In theory, if continuously activated, $70 \mathrm{ml}$ of BAT could clear approximately 20-30 g of glucose from the circulation over 24 hours (20). These results support a physiologically significant role for BAT in glucose homeostasis and insulin sensitivity in humans and also support the notion that BAT may function as an antidiabetic tissue in humans. Because variable observations from several studies appear to be due to different experimental protocols, such as temperature conditions and duration of cold exposure, future studies will need to establish standardized experimental protocols and exclusion criteria for patients taking medications, in particular, antidiabetic medications (115).

\section{Emerging questions in human BAT biology}

Recent studies indicate that adult human BAT is recruitable and contains beige-like adipocytes. This makes beige adipocytes a fascinating therapeutic target for obesity and diabetes in adult humans. It is important to note that human brown adipocytes possess a unique molecular signature, even though they resemble beige adipocytes, such that functions or effects of new regulatory factors/compounds should be critically evaluated in human cells. In the past, numbers of pharmacologic agents have been tested as antiobesity medications to activate BAT thermogenesis through the $\beta A R$ pathway. However, such attempts eventually failed, partially due to a lack of efficacy in obese populations and to adverse effects, which included elevated blood pressure and heart rate (116). Better understanding of the beige adipocyte-selective signaling pathways will provide new insights that will allow this cell type to be targeted. A plausible approach is to perform unbiased chemical compound screening to dissect the selective signaling pathways that control brown and/or beige adipocytes. To this end, we have recently developed an in vivo model, ThermoMouse, in which luciferase expression faithfully recapitulates endogenous UCP1 expression (117). This tool provides new opportunities to identify pharmacologic modulators of brown and beige adipocyte development.

Clearly, new technologies are needed to assess the efficacy of therapeutic agents on BAT mass and function in adult humans. ${ }^{18} \mathrm{~F}-\mathrm{FDG}-\mathrm{PET} / \mathrm{CT}$ scans measure glucose uptake (i.e., activated BAT mass) but do not accurately measure total BAT mass per se. MRI scans may circumvent some issues, such as high radiation exposure from ${ }^{18} \mathrm{~F}-\mathrm{FDG}-\mathrm{PET} / \mathrm{CT}$ scan, but MRI scanning may not be a realistic tool for screening purposes. Additionally, currently available devices do not have enough sensitivity and resolution to detect UCP1-positive adipocytes that sporadically reside in subcutaneous WAT and other adipose depots. Searching for brown/beige-selective cell surface markers or circulating biomarkers may lead to new methodologies that overcome such hurdles.

Last, it will be important to investigate mechanisms of BATmediated regulation of systemic and peripheral glucose homeostasis. Accumulating evidence suggests that BAT is not simply a heat-generating organ but also has an immense impact on systemic and peripheral glucose homeostasis by functioning as a metabolic sink for glucose, triglycerides, and possibly some metabolites. As discussed earlier, a number of endocrine factors mediate the link between BAT and peripheral organs, such as skeletal muscle and liver. Understanding BAT-mediated interorgan communication would further uncover new roles for BAT in energy and glucose homeostasis.

\section{Acknowledgments}

Because of space limitations, we apologize for the inability to cite a vast number of papers that contributed to the development of this field. This work was supported by grants from the NIH (DK087853 and DK97441) to S. Kajimura and grants from the NIH (P50-GM60338 and Clinical and Translational Science Award [UL1TR000071]), Shriners Hospitals for Children (85310 and 84090), and American Diabetes Association (1-14-TS-35) to L. Sidossis. We acknowledge support from the Pew Charitable 
Trust and PRESTO, Japan Science and Technology Agency, to S. Kajimura and the Sealy Center on Aging at University of Texas, Medical Branch at Galveston, to L. Sidossis.

Address correspondence to: Labros Sidossis, Shriners Hospitals for Children, Galveston, 815 Market Street, Rm 618, Gal- veston, Texas 77555-0177, USA. Phone: 409.770.6819; E-mail: lasidoss@utmb.edu. Or to: Shingo Kajimura, UCSF Diabetes Center, Department of Cell and Tissue Biology, UCSF, 35 Medical Center Way, Regeneration Medicine Building 1023, Box 0669, San Francisco, California 94143-0669, USA. Phone: 415.476.9644; E-mail: skajimura@diabetes.ucsf.edu.
1. Cunningham DJ, Stolwijk JA, Wenger CB. Comparative thermoregulatory responses of resting men and women. J Appl Physiol. 1978;45(6):908-915.

2. Lean ME. Brown adipose tissue in humans. Proc Nutr Soc. 1989;48(2):243-256.

3. Bonnot E. The interscapular gland. J Anat Physiol. 1908;43(pt 1):43-58.

4. Rasmussen A. The glandular status of brown multilocular adipose tissue. Endocrinology. 1922;6(6):760-770.

5. Huttunen P, Kortelainen ML. Long-term alcohol consumption and brown adipose tissue in man. Eur JAppl Physiol Occup Physiol. 1990;60(6):418-424.

6. Huttunen P, Hirvonen J, Kinnula V. The occurrence of brown adipose tissue in outdoor workers. Eur J Appl Physiol Occup Physiol. 1981;46(4):339-345.

7. Heaton JM. The distribution of brown adipose tissue in the human. J Anat. 1972;112(pt 1):35-39.

8. Astrup A, Bulow J, Christensen NJ, Madsen J. Ephedrine-induced thermogenesis in man: no role for interscapular brown adipose tissue. Clin Sci (Lond). 1984;66(2):179-186.

9. Hany TF, Gharehpapagh E, Kamel EM, Buck A, Himms-Hagen J, von Schulthess GK. Brown adipose tissue: a factor to consider in symmetrical tracer uptake in the neck and upper chest region. Eur J Nucl Med Mol Imaging. 2002;29(10):1393-1398.

10. Yeung HW, Grewal RK, Gonen M, Schoder H, Larson SM. Patterns of (18)F-FDG uptake in adipose tissue and muscle: a potential source of false-positives for PET. J Nucl Med. 2003;44(11):1789-1796.

11. Truong MT, et al. Focal FDG uptake in mediastinal brown fat mimicking malignancy: a potential pitfall resolved on PET/CT. AJR Am J Roentgenol. 2004;183(4):1127-1132.

12. Soderlund V, Larsson SA, Jacobsson H. Reduction of FDG uptake in brown adipose tissue in clinical patients by a single dose of propranolol. Eur J Nucl Med Mol Imaging. 2007;34(7):1018-1022.

13. Christensen CR, Clark PB, Morton KA. Reversal of hypermetabolic brown adipose tissue in F-18 FDG PET imaging. Clin Nucl Med. 2006;31(4):193-196.

14. Garcia CA, et al. Reduction of brown fat 2-deoxy2-[F-18]fluoro-D-glucose uptake by controlling environmental temperature prior to positron emission tomography scan. Mol Imaging Biol. 2006;8(1):24-29.

15. van Marken Lichtenbelt WD, et al. Cold-activated brown adipose tissue in healthy men. $N$ Engl J Med. 2009;360(15):1500-1508.

16. Cypess AM, et al. Identification and importance of brown adipose tissue in adult humans. $N$ EnglJ Med. 2009;360(15):1509-1517.

17. Virtanen KA, et al. Functional brown adipose tissue in healthy adults. N EnglJMed.
2009;360(15):1518-1525.

18. Nedergaard J, Bengtsson T, Cannon B. Unexpected evidence for active brown adipose tissue in adult humans. Am J Physiol Endocrinol Metab. 2007;293(2):E444-E452.

19. Saito M, et al. High incidence of metabolically active brown adipose tissue in healthy adult humans: effects of cold exposure and adiposity. Diabetes. 2009;58(7):1526-1531.

20. Chondronikola M, et al. Brown adipose tissue improves whole body glucose homeostasis and insulin sensitivity in humans. Diabetes. 2014;63(12):4089-4099.

21. Orava J, et al. Blunted metabolic responses to cold and insulin stimulation in brown adipose tissue of obese humans. Obesity (Silver Spring). 2013;21(11):2279-2287.

22. Kajimura S, Saito M. A new era in brown adipose tissue biology: molecular control of brown fat development and energy homeostasis. Annu Rev Physiol. 2014;76:225-249.

23. Harms M, Seale P. Brown and beige fat: development, function and therapeutic potential. Nat Med. 2013;19(10):1252-1263.

24. Wu J, Cohen P, Spiegelman BM. Adaptive thermogenesis in adipocytes: is beige the new brown? Genes Dev. 2013;27(3):234-250.

25. Shabalina IG, Petrovic N, de Jong JM, Kalinovich AV, Cannon B, Nedergaard J. UCP1 in brite/beige adipose tissue mitochondria is functionally thermogenic. Cell Rep. 2013;5(5):1196-1203.

26. Wu J, et al. Beige adipocytes are a distinct type of thermogenic fat cell in mouse and human. Cell. 2012;150(2):366-376.

27. Lidell ME, et al. Evidence for two types of brown adipose tissue in humans. Nat Med. 2013;19(5):631-634.

28. Cinti S. The Adipose Organ. Milano, Italy: Editrice Kurtis; 1999.

29. Murano I, Barbatelli G, Giordano A, Cinti S. Noradrenergic parenchymal nerve fiber branching after cold acclimatisation correlates with brown adipocyte density in mouse adipose organ. J Anat. 2009;214(1):171-178.

30. Zingaretti MC, et al. The presence of UCP1 demonstrates that metabolically active adipose tissue in the neck of adult humans truly represents brown adipose tissue. FASEB J. 2009;23(9):3113-3120.

31. Atit $\mathrm{R}$, et al. Beta-catenin activation is necessary and sufficient to specify the dorsal dermal fate in the mouse. Dev Biol. 2006;296(1):164-176.

32. Seale $P$, et al. PRDM16 controls a brown fat/skeletal muscle switch. Nature. 2008;454(7207):961-967.

33. Lepper C, Fan CM. Inducible lineage tracing of Pax7-descendant cells reveals embryonic origin of adult satellite cells. Genesis. 2010;48(7):424-436.

34. Walden TB, Timmons JA, Keller P, Nedergaard J, Cannon B. Distinct expression of muscle-specific microRNAs (myomirs) in brown adipocytes.
JCell Physiol. 2009;218(2):444-449.

35. Timmons JA, et al. Myogenic gene expression signature establishes that brown and white adipocytes originate from distinct cell lineages. Proc Natl Acad Sci U S A. 2007;104(11):4401-4406.

36. Forner F, Kumar C, Luber CA, Fromme T, Klingenspor M, Mann M. Proteome differences between brown and white fat mitochondria reveal specialized metabolic functions. Cell Metab. 2009;10(4):324-335.

37. Lee YH, Petkova AP, Mottillo EP, Granneman JG. In vivo identification of bipotential adipocyte progenitors recruited by beta3-adrenoceptor activation and high-fat feeding. Cell Metab. 2012;15(4):480-491.

38. Sanchez-Gurmaches J, Hung CM, Sparks CA, Tang Y, Li H, Guertin DA. PTEN loss in the Myf5 lineage redistributes body fat and reveals subsets of white adipocytes that arise from Myf5 precursors. Cell Metab. 2012;16(3):348-362.

39. Long JZ, et al. A smooth muscle-like origin for beige adipocytes. Cell Metab. 2014;19(5):810-820.

40. Barbatelli G, et al. The emergence of cold-induced brown adipocytes in mouse white fat depots is determined predominantly by white to brown adipocyte transdifferentiation. Am JPhysiol Endocrinol Metab. 2010;298(6):E1244-E1253.

41. Himms-Hagen J, Melnyk A, Zingaretti MC, Ceresi E, Barbatelli G. Multilocular fat cells in WAT of CL-316243-treated rats derive directly from white adipocytes. Am J Physiol Cell Physiol. 2000;279(3):C670-C681.

42. Frontini A, et al. White-to-brown transdifferentiation of omental adipocytes in patients affected by pheochromocytoma. Biochim Biophys Acta. 2013;1831(5):950-959.

43. Rosenwald M, Perdikari A, Rülicke T, Wolfrum C. Bi-directional interconversion of brite and white adipocytes. Nat Cell Biol. 2013;15(6):659-667.

44. Wang QA, Tao C, Gupta RK, Scherer PE. Tracking adipogenesis during white adipose tissue development, expansion and regeneration. Nat Med. 2013;19(10):1338-1344.

45. Sharp LZ, et al. Human BAT possesses molecular signatures that resemble beige/brite cells. PLOS One. 2012;7(11):e49452.

46. Petrovic N, Walden TB, Shabalina IG, Timmons JA, Cannon B, Nedergaard J. Chronic peroxisome proliferator-activated receptor gamma (PPAR $\gamma$ ) activation of epididymally derived white adipocyte cultures reveals a population of thermogenically competent, UCP1-containing adipocytes molecularly distinct from classic brown adipocytes. J Biol Chem. 2009;285(10):7153-7164.

47. Jespersen NZ, et al. A classical brown adipose tissue mRNA signature partly overlaps with brite in the supraclavicular region of adult humans. Cell Metab. 2013;17(5):798-805.

48. Cypess AM, et al. Anatomical localization, gene 
expression profiling and functional characterization of adult human neck brown fat. Nat Med. 2013;19(5):635-639.

49. Yoneshiro T, et al. Recruited brown adipose tissue as an antiobesity agent in humans. JClin Invest. 2013;123(8):3404-3408.

50. van der Lans AA, et al. Cold acclimation recruits human brown fat and increases nonshivering thermogenesis. JClin Invest. 2013;123(8):3395-3403.

51. Lee $P$, et al. Temperature-acclimated brown adipose tissue modulates insulin sensitivity in humans. Diabetes. 2014;63(11):3686-3698.

52. Kajimura S, Seale P, Spiegelman BM. Transcriptional control of brown fat development. Cell Metab. 2010;11(4):257-262.

53. Collins S. $\beta$-Adrenoceptor signaling networks in adipocytes for recruiting stored fat and energy expenditure. Front Endocrinol (Lausanne). 2011;2:102.

54 . Nguyen KD, et al. Alternatively activated macrophages produce catecholamines to sustain adaptive thermogenesis. Nature. 2011;480(7375):104-108.

55. Qiu Y, et al. Eosinophils and type 2 cytokine signaling in macrophages orchestrate development of functional beige fat. Cell. 2014;157(6):1292-1308.

56. Cao L, et al. White to brown fat phenotypic switch induced by genetic and environmental activation of a hypothalamic-adipocyte axis. Cell Metab. 2011;14(3):324-338.

57. Bostrom P, et al. A PGC1- $\alpha$-dependent myokine that drives brown-fat-like development of white fat and thermogenesis. Nature. 2012;481(7382):463-468.

58. Xu X, et al. Exercise ameliorates high-fat dietinduced metabolic and vascular dysfunction, and increases adipocyte progenitor cell population in brown adipose tissue. Am J Physiol Regul Integr Comp Physiol. 2011;300(5):R1115-R1125.

59. Finck BN, Kelly DP. PGC-1 coactivators: inducible regulators of energy metabolism in health and disease. J Clin Invest. 2006;116(3):615-622.

60. Rao RR, et al. Meteorin-like is a hormone that regulates immune-adipose interactions to increase beige fat thermogenesis. Cell. 2014;157(6):1279-1291.

61. Lee P, et al. Irisin and FGF21 are cold-induced endocrine activators of brown fat function in humans. Cell Metab. 2014;19(2):302-309.

62. Knudsen JG, et al. Role of IL- 6 in exercise training- and cold-induced UCP1 expression in subcutaneous white adipose tissue. PLoS One. 2014;9(1):e84910.

63. Carriere A, et al. Browning of white adipose cells by intermediate metabolites: an adaptive mechanism to alleviate redox pressure. Diabetes. 2014;63(10):3253-3265.

64. Petruzzelli M, et al. A switch from white to brown fat increases energy expenditure in cancer-associated cachexia. Cell Metab. 2014;20(3):433-447.

65. Kir S, et al. Tumour-derived PTH-related protein triggers adipose tissue browning and cancer cachexia. Nature. 2014;513(7516):100-104.

66. Qian SW, et al. BMP4-mediated brown fat-like changes in white adipose tissue alter glucose and energy homeostasis. Proc Natl Acad Sci U S A. 2013;110(9):E798-E807.

67. Schulz TJ, et al. Identification of inducible brown adipocyte progenitors residing in skeletal muscle and white fat. Proc Natl Acad Sci US A.
2011;108(1):143-148.

68. Tseng YH, et al. New role of bone morphogenetic protein 7 in brown adipogenesis and energy expenditure. Nature. 2008;454(7207):1000-1004.

69. Whittle AJ, et al. BMP8B increases brown adipose tissue thermogenesis through both central and peripheral actions. Cell. 2012;149(4):871-885.

70. Tomlinson E, et al. Transgenic mice expressing human fibroblast growth factor-19 display increased metabolic rate and decreased adiposity. Endocrinology. 2002;143(5):1741-1747.

71. Fu L, et al. Fibroblast growth factor 19 increases metabolic rate and reverses dietary and leptin-deficient diabetes. Endocrinology. 2004;145(6):2594-2603.

72. Fisher FM, et al. FGF21 regulates PGC-1 $\alpha$ and browning of white adipose tissues in adaptive thermogenesis. Genes Dev. 2012;26(3):271-281.

73. Emanuelli B, et al. Interplay between FGF21 and insulin action in the liver regulates metabolism. J Clin Invest. 2014;124(2):515-527.

74. Hinoi E, et al. Growth differentiation factor-5 promotes brown adipogenesis in systemic energy expenditure. Diabetes. 2014;63(1):162-175.

75. Bordicchia M, et al. Cardiac natriuretic peptides act via p38 MAPK to induce the brown fat thermogenic program in mouse and human adipocytes. J Clin Invest. 2012;122(3):1022-1036.

76. Madsen L, et al. UCP1 induction during recruitment of brown adipocytes in white adipose tissue is dependent on cyclooxygenase activity. PLOS One. 2010;5(6):e11391.

77. Vegiopoulos A, et al. Cyclooxygenase-2 controls energy homeostasis in mice by de novo recruitment of brown adipocytes. Science. 2010;328(5982):1158-1161.

78. Sun K, et al. Dichotomous effects of VEGF-A on adipose tissue dysfunction. Proc Natl Acad Sci U S A. 2012;109(15):5874-5879.

79. Elias I, et al. Adipose tissue overexpression of vascular endothelial growth factor protects against diet-induced obesity and insulin resistance. Diabetes. 2012;61(7):1801-1813.

80. Roberts LD, et al. beta-Aminoisobutyric acid induces browning of white fat and hepatic $\beta$-oxidation and is inversely correlated with cardiometabolic risk factors. Cell Metab. 2014;19(1):96-108

81. Yadav $\mathrm{H}$, et al. Protection from obesity and diabetes by blockade of TGF- $\beta /$ Smad3 signaling. Cell Metab. 2011;14(1):67-79.

82. Koncarevic A, et al. A novel therapeutic approach to treating obesity through modulation of TGF $\beta$ signaling. Endocrinology. 2012;153(7):3133-3146.

83. Fournier B, et al. Blockade of the activin receptor IIb activates functional brown adipogenesis and thermogenesis by inducing mitochondrial oxidative metabolism. Mol Cell Biol. 2012;32(14):2871-2879.

84. Kiefer FW, et al. Retinaldehyde dehydrogenase 1 regulates a thermogenic program in white adipose tissue. Nat Med. 2012;18(6):918-925.

85. Bi P, et al. Inhibition of Notch signaling promotes browning of white adipose tissue and ameliorates obesity. Nat Med. 2014;20(8):911-918.

86. Kajimura S, et al. Initiation of myoblast to brown fat switch by a PRDM16-C/EBP- $\beta$ transcriptional complex. Nature. 2009;460(7259):1154-1158.

87. Kajimura S, et al. Regulation of the brown and white fat gene programs through a PRDM16/
CtBP transcriptional complex. Genes Dev. 2008;22(10):1397-1409.

88. Ohno H, Shinoda K, Ohyama K, Sharp LZ, Kajimura S. EHMT1 controls brown adipose cell fate and thermogenesis through the PRDM16 complex. Nature. 2013;504(7478):163-167.

89. Harms MJ, et al. Prdm16 is required for the maintenance of brown adipocyte identity and function in adult mice. Cell Metab. 2014;19(4):593-604.

90. Rajakumari S, et al. EBF2 determines and maintains brown adipocyte identity. Cell Metab. 2013;17(4):562-574.

91. Ohno H, Shinoda K, Spiegelman BM, Kajimura S. PPAR $\gamma$ agonists induce a white-to-brown fat conversion through stabilization of PRDM16 protein. Cell Metab. 2012;15(3):395-404.

92. Cohen P, et al. Ablation of PRDM16 and beige adipose causes metabolic dysfunction and a subcutaneous to visceral fat switch. Cell. 2014;156(1):304-316

93. Kozak LP. The genetics of brown adipocyte induction in white fat depots. Front Endocrinol (Lausanne). 2011;2:64.

94. Xue B, Rim JS, Hogan JC, Coulter AA, Koza RA, Kozak LP. Genetic variability affects the development of brown adipocytes in white fat but not in interscapular brown fat. J Lipid Res. 2007;48(1):41-51

95. Xue B, Coulter A, Rim JS, Koza RA, Kozak LP. Transcriptional synergy and the regulation of Ucp1 during brown adipocyte induction in white fat depots. Mol Cell Biol. 2005;25(18):8311-8322.

96. Valve R, Heikkinen S, Rissanen A, Laakso M, Uusitupa M. Synergistic effect of polymorphisms in uncoupling protein 1 and beta3-adrenergic receptor genes on basal metabolic rate in obese Finns. Diabetologia. 1998;41(3):357-361.

97. Nagai N, Sakane N, Tsuzaki K, Moritani T. UCP1 genetic polymorphism (-3826 A/G) diminishes resting energy expenditure and thermoregulatory sympathetic nervous system activity in young females. Int JObes (Lond). 2011;35(8):1050-1055

98. Yoneshiro T, et al. Impact of UCP1 and beta3AR gene polymorphisms on age-related changes in brown adipose tissue and adiposity in humans. Int J Obes (Lond). 2013;37(7):993-998.

99. Cormier-Daire V, et al. Cryptic terminal deletion of chromosome 9q34: a novel cause of syndromic obesity in childhood? JMed Genet. 2003;40(4):300-303.

100.Willemsen $\mathrm{MH}$, et al. Update on Kleefstra syndrome. Mol Syndromol. 2012;2(3):202-212.

101.Wang J, et al. Ablation of LGR4 promotes energy expenditure by driving white-to-brown fat switch. Nat Cell Biol. 2013;15(12):1455-1463.

102. Feldmann HM, Golozoubova V, Cannon B, Nedergaard J. UCP1 ablation induces obesity and abolishes diet-induced thermogenesis in mice exempt from thermal stress by living at thermoneutrality. Cell Metab. 2009;9(2):203-209.

103. Stanford KI, et al. Brown adipose tissue regulates glucose homeostasis and insulin sensitivity. J Clin Invest. 2013;123(1):215-223.

104. Bartelt A, et al. Brown adipose tissue activity controls triglyceride clearance. Nat Med. 2011;17(2):200-205.

105. Seale P, et al. Prdm16 determines the thermo- 
genic program of subcutaneous white adipose tissue in mice. JClin Invest. 2011;121(1):96-105.

106.Pfannenberg C, et al. Impact of age on the relationships of brown adipose tissue with sex and adiposity in humans. Diabetes. 2010;59(7):1789-1793.

107. Lee P, Greenfield JR, Ho KK, Fulham MJ. A critical appraisal of the prevalence and metabolic significance of brown adipose tissue in adult humans. Am J Physiol Endocrinol Metab. 2010;299(4):E601-E606.

108. Jacene HA, Cohade CC, Zhang Z, Wahl RL. The relationship between patients' serum glucose levels and metabolically active brown adipose tissue detected by PET/CT. Mol Imaging Biol. 2011;13(6):1278-1283.

109. Ouellet V, et al. Outdoor temperature, age, sex, body mass index, and diabetic status determine the prevalence, mass, and glucose-uptake activity of 18F-FDG-detected BAT in humans. JClin Endocrinol Metab. 2011;96(1):192-199.

110. Orava J, et al. Different metabolic responses of human brown adipose tissue to activation by cold and insulin. Cell Metab. 2011;14(2):272-279.

111. Chen KY, et al. Brown fat activation mediates cold-induced thermogenesis in adult humans in response to a mild decrease in ambient temperature. JClin Endocrinol Metab 2013;98(7):E1218-E1223.

112. Yoneshiro T, et al. Brown adipose tissue, wholebody energy expenditure, and thermogenesis in healthy adult men. Obesity. 2011;19(1):13-16.

113. Ouellet V, et al. Brown adipose tissue oxidative metabolism contributes to energy expenditure during acute cold exposure in humans. JClin Invest. 2012;122(2):545-552.

114. Matsushita M, Yoneshiro T, Aita S, Kameya T, Sugie $\mathrm{H}$, Saito M. Impact of brown adipose tissue on body fatness and glucose metabolism in healthy humans. Int JObes (Lond). 2014;38(6):812-817.

115. Cypess AM, Haft CR, Laughlin MR, Hu HH. Brown fat in humans: consensus points and experimental guidelines. Cell Metab. 2014;20(3):408-415.

116. Carey AL, et al. Ephedrine activates brown adipose tissue in lean but not obese humans. Diabetologia. 2013;56(1):147-155.

117. Galmozzi A, et al. ThermoMouse: An in vivo model to identify modulators of UCP1 expression in brown adipose tissue. Cell Report. 2014;9(5):1-10. 